China Ocean Eng., 2021, Vol. 35, No. 6, P. 802-813

DOI: https://doi.org/10.1007/s13344-021-0071-7, ISSN 0890-5487

http://www.chinaoceanengin.cn/ E-mail: coe@nhri.cn

\title{
Optimal Port Design Minimizing Standing Waves with A Posteriori Long Term Shoreline Sustainability Analysis
}

\author{
Megan COOK ${ }^{\mathrm{a}, \mathrm{b}, \mathrm{c}, \text { * }}$, Frédéric BOUCHETTE, c, Bijan MOHAMMADI ${ }^{\mathrm{c}, \mathrm{d}}$, Léa SPRUNCK ${ }^{\mathrm{b}, \mathrm{c}}$, \\ Nicolas FRAYSSE ${ }^{\mathrm{d}}$ \\ a BRL Ingénierie, Nîmes 30000, France \\ b GEOSCIENCES-M, University of Montpellier, CNRS, Montpellier 34090, France \\ ${ }^{c}$ GLADYS, University of Montpellier, CNRS, Le Grau du Roi 30240, France \\ d IMAG, University of Montpellier, CNRS, Montpellier 34090, France
}

Received April 20, 2021; revised August 11, 2021; accepted September 20, 2021

(C2021 Chinese Ocean Engineering Society and Springer-Verlag GmbH Germany, part of Springer Nature

\begin{abstract}
Optimization theory is applied to a coastal engineering problem that is the design of a port. This approach was applied to the redesign of La Turballe Port in order to increase the exploitable surface area and simultaneously reduce the occurrence of long waves within the port. Having defined the cost function as a weighted function of wave amplitude and with the chosen parameterization of the port, results show that an extended jetty and a widened mole yield a unique optimal solution. This work demonstrates that numerical optimization may be quick and efficient in the identification of port solutions consistent with classic engineering even in the context of complex problems.
\end{abstract}

Key words: optimization, coastal engineering, harbour design, hydrodynamics, shoreline analysis

Citation: Megan, C., Frédéric, B., Bijan, M., Léa, S., Nicolas, F., 2021. Optimal port design minimizing standing waves with a posteriori long term shoreline sustainability analysis. China Ocean Eng., 35(6): 802-813, doi: https://doi.org/10.1007/s13344-021-0071-7

\section{Introduction}

When designing a port, an extensive study should be conducted to ensure a smooth functioning of services, improve the experience of its users, and provide sufficient protection of the port. This study on the port, its structural components and its users should combine different approaches such as risk identification methods and extensive surveys of the site (Joubert and Pretorius, 2020; Trbojevic and Carr, 2000; Alises et al., 2014; McCartney, 1985; Hewitt and Martin, 2001), the development of design criteria on the different components of the harbour (Sawicki et al., 1998; Jahren and Ishii, 1995; Smith, 1988; Allsop and Hettiarachchi, 1989) and numerical and physical simulations (Wang et al., 2015, 2017; Tian et al., 2018; Lillycrop and Briggs, 1992; Groenveld, 1983; Pachakis and Kiremidjian, 2003; Cornett and Baker, 2018; Harris et al., 2009). We present a method by optimization to supplement these standard procedures.

The term optimization in the field of coastal dynamics refers to the transformation of the natural seabed or the geometric and rheological properties of artificial structures present in ports or at the coast and leads to the minimization of a scalar quantity. This quantity, named cost function and denoted $\mathscr{G}$, is representative of the state of the system and is generally associated with certain physical quantities, such as those related to waves or currents. Applications of optimization theory to coastal dynamics already exist in literature. The work by Isebe et al. (2008b) sought to minimize the $L^{2}$ norm of the water waves free surface elevation in the design of harbours or offshore breakwaters, and Isebe et al. (2008a) and Bouharguane et al. (2010) used this approach when designing coastal protection structures while minimizing the effect of beach erosion. Other examples of optimal design of coastal structures include $\mathrm{Wu}$ et al. (2018); Saghi et al. (2020); Li and Zhou (2015); Zhang et al. (2016); Castillo et al. (2004). Alternatively, Mohammadi and Bouharguane (Mohammadi and Bouharguane, 2011, 2014; Bouharguane and Mohammadi, 2012) used optimization theory as a tool in the modeling of the dynamics of seabeds in shallow waters. A general presentation of the methods used here can be found in Ivorra et al. (2005); Mohammadi and Pironneau (2009); Mohammadi and Saiac (2003), and we refer to these documents for the theoretical bases of optimization theory and its applications to coastal systems and coastal management.

It is indisputable that a model based on optimization the-

*Corresponding author. E-mail: megan.cook@umontpellier.fr 
ory can be an invaluable tool for the development or updating of port configurations. Ports and harbour are generally extended to accommodate the increasing number of commercial and economic activities. Ports are enlarged to increase their exploitable surface area. This increase can be achieved with a second objective in mind. For instance, as well as increasing the surface area, one may also wish to reduce the agitation of the water within the port by introducing additional port protection, using jetties, breakwaters and groins. However, the increase in exploitable surface area may cause an increase in agitation or, conversely, the decrease of wave agitation may cause a decrease in surface area. Therefore, the design of a port has no trivial solution. Additional difficulties include the large number of geometric transformations considered in such a study; therefore, employing a classic engineering approach could be difficult and time-consuming, requiring a large number of exploratory numerical simulations forced by a large range of different weather and sea conditions.

The work presented here was prompted by the desire to further the investigation in the redesign of ports via the introduction of optimal theory. The introduction of optimal theory in a management port operation originates from a successful operation that consisted in the deployment on the coast of Sète (France) of a geotube (Isebe et al., 2008c).

With the intention of accompanying the engineers with their analysis on harbor protection, we devise a tool, based on the concept of port agitation minimization and capable of identifying pertinent port configurations. This work was conducted in the case of La Turballe Port (France), but is valid for any semi-enclosed harbour with relatively constant water depth. This tool should in no way substitute the classical approach to port design performed by the experts in the field but is intended to offer suggestions on possible configurations. Oftentimes, numerical modeling of port configurations is costly, both in time and resources, and consequently, the number of simulations is restricted. The tool presented here offers a rapid, cost-efficient, and userfriendly resource designed to assist traditional approaches. This optimization model is capable of indicating optimal harbor designs in very little time, with regards to a predetermined objective function. Modifying the objective function, forcing conditions, and/or parameters surrounding the optimization model allows users to explore different harbor configurations in a short span of time. The resulting port designs can, and should, be subject to further investigation so as to evaluate their effectiveness, this being achieved using a more classical engineering approach. This tool also allows users to give credence to their initial hypotheses and may provide unorthodox results not necessarily envisioned by the experts.

This paper presents one such study where the focus is on the minimization of long wave energy within La Turballe Port, situated in North-Western France. The parameters are chosen with the aim of depicting a worst-case scenario.

We begin this paper with the hydrodynamic model used in the minimization of port agitation. Here, we use a variation of the Helmholtz equation applied to a port configuration because the control of wave/wave interactions, seiches and resonance phenomena is preeminent in the design of this inner port, in so far as gravity waves are efficiently fully dissipated at the entrance. Obviously, the hydrodynamic model must deal with different forcing conditions and returns water oscillation amplitudes. The next section is devoted to the concept of optimization theory applied to coastal engineering, and more specifically to the extension of ports, with the main objective to increase the exploitable surface area and minimize the agitation of the water within the port. In the third section, we apply this theory to the practical case of La Turballe Port in North-Western France and a discussion of the long term shoreline sustainability can be found in the final section.

\section{Hydrodynamic model for port agitation}

In order to calculate the optimal port configuration with regards to the minimization of water oscillations within the port, a hydrodynamic model capable of modeling the relevant water level fluctuations is required. Generally, water level in ports can be driven by tide, very low frequency waves, infra-gravity waves, waves or wind waves depending upon the context. Here, we know from historical experience that classic gravity waves are efficiently attenuated at the port entrance, but may transfer part of their energy to the inner port in the form of seiches and various standing low frequency waves. In addition, the model could take into account the different weather and deep sea scenarios observed off the coast, as well as the absorption/reflection characteristics of the structures within the harbour.

The model can be applied to any port verifying the required conditions (semi-enclosed basin with a forcing boundary and roughly constant water depth). We illustrate this model on the case of the old port of La Rochelle, as a generic port. This example was chosen because it illustrates the three different boundary conditions which we are able to consider with this model. The realistic application that follows in Section 4 does not present all the boundary conditions presented here, so hasn't been used as an illustration.

The model was designed with the application of $\mathrm{La}$ Turballe in mind (see Section 4), that is a port with input waves arriving with no angle of incidence.

\subsection{Settings}

We define the domain $\Omega$ as the area occupied by water within the port. The domain is a closed, compact subdomain of $\mathbb{R}^{2}$. We denote $\Lambda$ the boundary of $\Omega$, which is to be partitioned into three subsets $\left(\Lambda_{\mathrm{i}}, \Lambda_{\mathrm{w}}, \Lambda_{\mathrm{o}}\right)$. We denote $\Lambda_{\mathrm{i}}$ the boundary over which the incoming forcing is present. Forcing is given in the form of wave agitation at the entrance of 
the port, where the influx of energy arises. We denote $\Lambda_{\mathrm{W}}$ the borders of the domain representing the walls of the port. These walls are associated with an absorption/reflection coefficient in order to take into account the behavior of the waves when they reach the solid borders of the port. Finally, we denote $\Lambda_{\mathrm{o}}$ the boundary of $\Omega$ over which an outflux of energy is observed. This occurs when an outlet is present in the port, as is the case of the old port of La Rochelle (France). The different types of boundaries of $\Omega$ are illustrated in Fig. 1.

Port agitation is directly controlled by waves appearing at its entrance at $\Lambda_{\mathrm{i}}$, and no influx of energy is possible from the other borders; the solid structures forming the boundary $\Lambda_{\mathrm{w}}$ are considered impassible and no exchange of energy can cross it. Furthermore, no energy can appear from the outflux boundary $\Lambda_{\mathrm{o}}$. As such, the hydrodynamic model we consider is limited to the inside of the port with local forcings at its entrance.

Some ports, and especially the one at La Turballe, are known to be controlled by low frequency oscillations or seiches (Rabinovich, 2017) driven solely by the energy provided at the port entrance and the geometry of the port. Therefore, a model based on the Helmholtz equations is more suitable to describe the hydrodynamic processes within the port than a theory based on wave propagation.

\subsection{Helmholtz model}

For simplicity, we place ourselves in the setting of linear wave theory, which provides a first order description of the gravity waves on the surface of the water.

Similar to Isebe et al. (2008b), we consider a surface wave to be the sum of an incident wave and a reflected wave:

$\eta=\eta_{\mathrm{i}}+\eta_{\mathrm{r}}$.

The incident wave $\eta_{\mathrm{i}}$ is simply defined by: $\eta_{\mathrm{i}}(x, t)=a_{\mathrm{i}}(x) \mathrm{e}^{-\mathrm{i} \sigma t}$,

where the spatial component $a_{\mathrm{i}}$ is defined by:

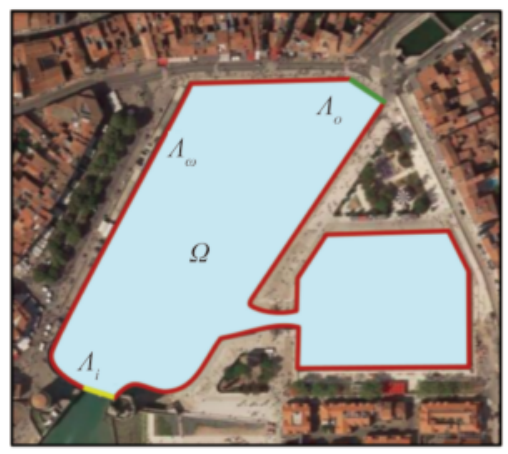

$a_{\mathrm{i}}(x)=a_{\max } \mathrm{e}^{-\mathrm{i} k x}$,

where, $\sigma$ is the wave frequency, $a_{\max }$ is the amplitude of the water surface oscillation for this given frequency, and $\boldsymbol{k}=k(\cos \theta, \sin \theta)$ is a wave number vector, with $k$ the wave number and $\theta$ the angle of propagation of the wave. The wave number $k$ is calculated over the domain using the linear dispersion equation (4). Practically, a shallow water approximation can be used as port standing waves are of very significant wave length with respect to mean water depth; $h$ is the water depth and $g$ is the gravitational acceleration.

$\sigma^{2}=g k \tanh (k h)$.

The reflected wave is defined by:

$\eta_{\mathrm{r}}(x, t)=a_{\mathrm{r}}(x) \mathrm{e}^{-\mathrm{i} \sigma t}$.

We suppose the spatial component $a_{\mathrm{r}}$ of the reflected wave satisfies the following Berkhoff equation (Berkhoff, 1972):

$\nabla \cdot\left(C C_{\mathrm{g}} \nabla a\right)+\sigma^{2} \frac{C_{\mathrm{g}}}{C} a=0$,

where $C$ and $C_{\mathrm{g}}$ are respectively the phase velocity and the group velocity of the wave.

Assuming constant depth within the port and $C_{\mathrm{g}}=\frac{1}{2} C$, and noting that $C=\frac{\sigma}{k}$, Eq. (6) can be simplified to yield the following Helmholtz equation:

$k^{2} a+\Delta a=0$.

\subsection{Eigen mode and domain resonance}

Solving Helmholtz equation can also be seen as looking for the eigen modes of the Laplace operator in the domain $\Omega$ under some boundary conditions:

$-\Delta u_{\lambda}=\lambda u_{\lambda}+b . c$.

where $\left(\lambda(\Omega), u_{\lambda}(\Omega)\right)$ are the eigen couple functions of the domain $\Omega$ which is variable in a context of shape optimization.

In situation where $|\lambda(\Omega)|=\|\boldsymbol{k}\|^{2}$, the solution of Eq. (8)

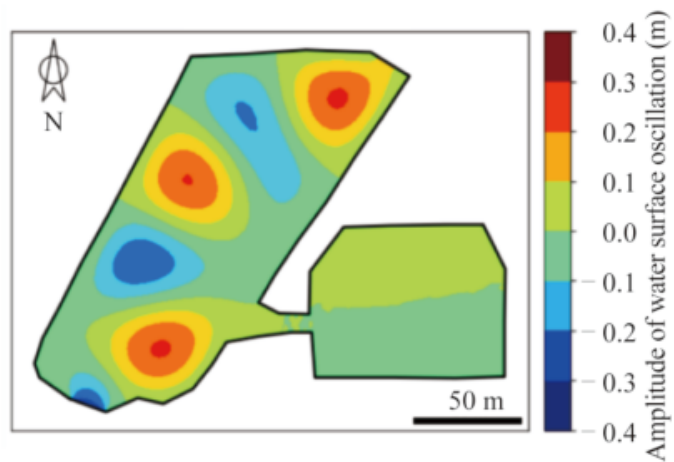

Fig. 1. Left: Illustration of the domain $\Omega$ and its borders on the old port of La Rochelle (France). The red borders depict the walls of the port, characterized by absorption and reflection. The yellow border indicates an influx of energy, depending on outer wave conditions. The green border indicates an outflux of energy, where the energy flux driven by dynamics of the water surface exits the port domain through the existing channel. Right: Numerical result of the hydrodynamic model applied to the La Rochelle configuration. The plot represents the resulting total wave field $a=a_{\mathrm{i}}+a_{\mathrm{r}}$ forced by incoming fairweather waves at an angle $110^{\circ}$ through $\Lambda_{\mathrm{i}}$, and fully transformed to standing long waves within the port. 
is an eigenmode of the Laplace operator. Exciting therefore this mode by a relevant incoming wave forcing will result in an infinite increase of the wave agitation in the basin and subsequently of the corresponding energy. We will address how to deal efficiently with this issue in the optimization section.

\subsection{Boundary conditions}

Boundary conditions need to be associated with Eq. (7) for the reflected wave field $a_{\mathrm{r}}$.

On forcing boundaries $\Lambda_{\mathrm{i}}$, the total wave field is composed solely of the incident wave field since no reflection occurs. This yields $\eta=\eta_{\mathrm{i}}$ and therefore $a_{\mathrm{r}}=0$ over $\Lambda_{\mathrm{i}}$.

On solid borders $\Lambda_{\mathrm{w}}$, a certain portion of the energy of the waves is reflected/absorbed when contact with this boundary is made. This reflective property is directly linked to the physical characteristics of the boundary. In the case of vertical rigid walls, almost all of the energy is reflected. On mild slope boundaries, more energy is absorbed/dissipated; the reflected wave field on these boundaries is equal to a portion of the incident wave field. Over $\Lambda_{\mathrm{w}}$, we have $\eta_{\mathrm{r}}=-\gamma \eta_{\mathrm{i}}$, where $\gamma \in[0,1]$ is a reflection/absorption coefficient. For $\gamma=1$, the border shows total reflection and $\gamma=0$ the border shows total absorption. This also yields $a_{\mathrm{r}}=-\gamma a_{\mathrm{i}}$ over $\Lambda_{\mathrm{w}}$.

Outlet borders have no direct influence of the wave field within $\Omega$, but energy can leave the domain via this boundary. We therefore apply the homogeneous Neumann boundary condition over $\Lambda_{\mathrm{o}}: \nabla a_{\mathrm{r}} \cdot \boldsymbol{n}=0$, where $\boldsymbol{n}$ is the outer unit normal and $\cdot$ represents the inner product operator.

\subsection{Numerical strategy}

The incident wave field is calculated analytically using Eq. (3), whereas the reflected wave field satisfies:

$$
\begin{cases}k^{2} a_{\mathrm{r}}+\Delta a_{\mathrm{r}}=0 & \text { over } \Omega \\ a_{\mathrm{r}}=0 & \text { on } \Lambda_{\mathrm{i}} \\ a_{\mathrm{r}}=-\gamma a_{\mathrm{i}} & \text { on } \Lambda_{\mathrm{w}} \\ \nabla a_{\mathrm{r}} \cdot \boldsymbol{n}=0 & \text { over } \Lambda_{\mathrm{o}}\end{cases}
$$

This time-independent elliptic partial differential equation which includes Neumann and Dirichlet boundary conditions is solved using a finite element method. This method was chosen because it allows the use of irregular grids with elements of different sizes and geometries, as well as the possibility of mesh adaptation. Given that a port may present intricate details and complex boundaries, a finite element method was a natural choice. Such a finite element method requires a weak formulation of the considered problem. In our case, the weak formulation of the Helmholtz problem (9) reads: find $a_{\mathrm{r}}$ such that

$\int_{\Omega} k^{2} a_{\mathrm{r}} v=\int_{\Omega} \nabla a_{\mathrm{r}} \nabla v+\int_{\Lambda_{\mathrm{w}}} \gamma\left(\nabla a_{\mathrm{i}} \cdot \boldsymbol{n}\right) v$

for all test functions $v$ of the same nature as $a_{\mathrm{r}}$. We use piecewise linear finite element functions to numerically de- termine the solution of Eq. (10) over a triangular adapted mesh.

Fig. 1 illustrates the resulting field of the water surface oscillations within the La Rochelle Port with the boundary conditions described previously and in the case of fair weather incoming conditions. We observe the arrival of energy over the forcing boundary $\Lambda_{\mathrm{i}}$ as well as the presence of bumps and nodes within the port resulted from the interactions of wave oscillations with the boundary walls. We also observe an output of energy at $\Lambda_{\mathrm{o}}$ where the energy leaves the domain $\Omega$ of the port and enters the adjoining canal.

\section{Optimal design model}

Using the previously described hydrodynamic model, we consider the following optimization approach in the design of ports based on agitation minimization.

\subsection{Application to port configuration}

We set $\psi$ as the set of parameters defining the possible transformations of the port. Examples include the dimensions of a groin, the angle of a jetty or width of the entrance. The values of these parameters are modifiable and are used to determine the optimal configuration of the port with regard to the minimization of the cost function. Given that $\psi$ determines the shape of the port, it is clear that the domain $\Omega$ varies in relation to $\psi$. Therefore, the computational domain of the hydrodynamic model changes at each step of the optimization method.

The optimization problem consists of minimizing the global agitation of long waves within the port while taking into account the different forcing scenarios that can be observed. The design of the port should reduce long wave agitation whether in the presence of fair weather or storms offshore. It is clear that priority should be given to the minimization of severe wave conditions over calmer ones, since greater damage is observed under stormy conditions. In this study, the frequency of occurrence of a given wave scenario is discarded and instead we focus on the minimization of waves of larger amplitude. Let $N$ be the total number of forcing scenarios considered in the study of the port design. We associate each scenario with an index $n \in\{0, \ldots, N\}$. In order to incorporate these different forcing scenarios, we define the local cost functions $\mathscr{I}_{n}$ associated with a given scenario $n$ and combine them in a purposeful manner to form the global function $\mathcal{G}$ to be minimized.

\subsection{Choice of cost function}

For a given forcing scenario $n$, we consider the following local cost function:

$$
\mathscr{I}_{n}(\psi)=\frac{1}{K(\mathscr{P})} \frac{1}{|\Omega(\psi)|} \int_{\Omega(\psi)} \mathscr{E}_{n}(\psi, \boldsymbol{x}) \mathscr{P}(\boldsymbol{x}) \mathrm{d} \boldsymbol{x},
$$

where $\psi$ is the parameterization of the port modifiable in the search of an optimal configuration and $|\Omega(\psi)|$ is the surface area of $\Omega(\psi)$. The quantity $\mathscr{E}_{n}(\psi, x)=\frac{1}{2} \rho g\left[a_{n}(\psi, x)\right]^{2}$ is the 
total surface energy defined over the domain $\Omega$ and associated with the forcing scenario $n$ and the configuration $\psi$ of the port. Here, $\rho$ is the density of the water, $g$ is the gravitational acceleration and $a_{n}(\psi)$ the amplitude of the waves calculated over $\Omega(\psi)$ associated with the forcing scenario $n$ and the configuration $\psi$ of the port. The function $\mathscr{P}$, named spatial weight function, enables us to prioritize the minimization of the agitation over certain preferred zones of the port, with $K(\mathscr{P}, \Omega(\psi))=\int_{\Omega(\psi)} \mathscr{P}(\boldsymbol{x}) \mathrm{d} x$. In practice, these zones are defined by traditional port engineers in order to focus the minimization of agitation in zones nearby mooring stations for boats or in which high maritime circulation is expected. Examples of spatial weight functions include $\mathscr{P}_{0}(\boldsymbol{x})=1$, where no zone is prioritized over another, or alternatively

$$
\mathscr{P}_{1}(x, y)=\exp \left\{-A\left[\left(x-x_{A}\right)^{4}+\left(y-y_{A}\right)^{4}\right]\right\},
$$

where a circular zone within the port is considered a priority, the center of which is given by the coordinates $\left(x_{A}, y_{A}\right)$ and $A$ defines its radius.

The global cost function $\mathscr{G}$ is a linear combination of the local cost functions defined by Eq. (11), where the scalar coefficients are determined by the wave energy at the forcing boundary. Let $a_{\mathrm{i}}(n)$ be the amplitude of the incoming waves (defined on $\Lambda_{\mathrm{i}}$ ) associated with the forcing scenario $n$, and the global cost function to be minimized in the search of the optimal solution is defined as:

$$
\mathscr{g}(\psi)=\frac{\sum_{n=1}^{N} a_{\mathrm{i}}(n) \mathscr{g}_{n}(\psi)}{\sum_{n=1}^{N} a_{\mathrm{i}}(n)} \text {. }
$$

The choice of this global cost function ensures priority to be given to the minimization of the local cost functions associated with the more extreme weather scenarios. These scenarios have the ability to cause the most damage within the port, so it is natural to design a global cost function capable of prioritizing the more severe weather conditions.

\subsection{Numerical strategy}

Fig. 2 illustrates the numerical strategy put in place in the search of the optimal port configuration. The model explores the different port configurations allowed by the user. For each forcing scenario, wave agitation is calculated using the hydrodynamic model presented in Section 2. This includes calculating the incident wave analytically and solving the Helmholtz equation with a PDE solver for the reflected waves. The local cost function Eq. (11) can then be determined. Once all the local cost functions have been calculated, it is then possible to determine the global cost function $\mathscr{I}$ defined by Eq. (13). It is then possible to determine the optimal port configuration with regards to $\mathscr{Y}$, which concludes the simulation.

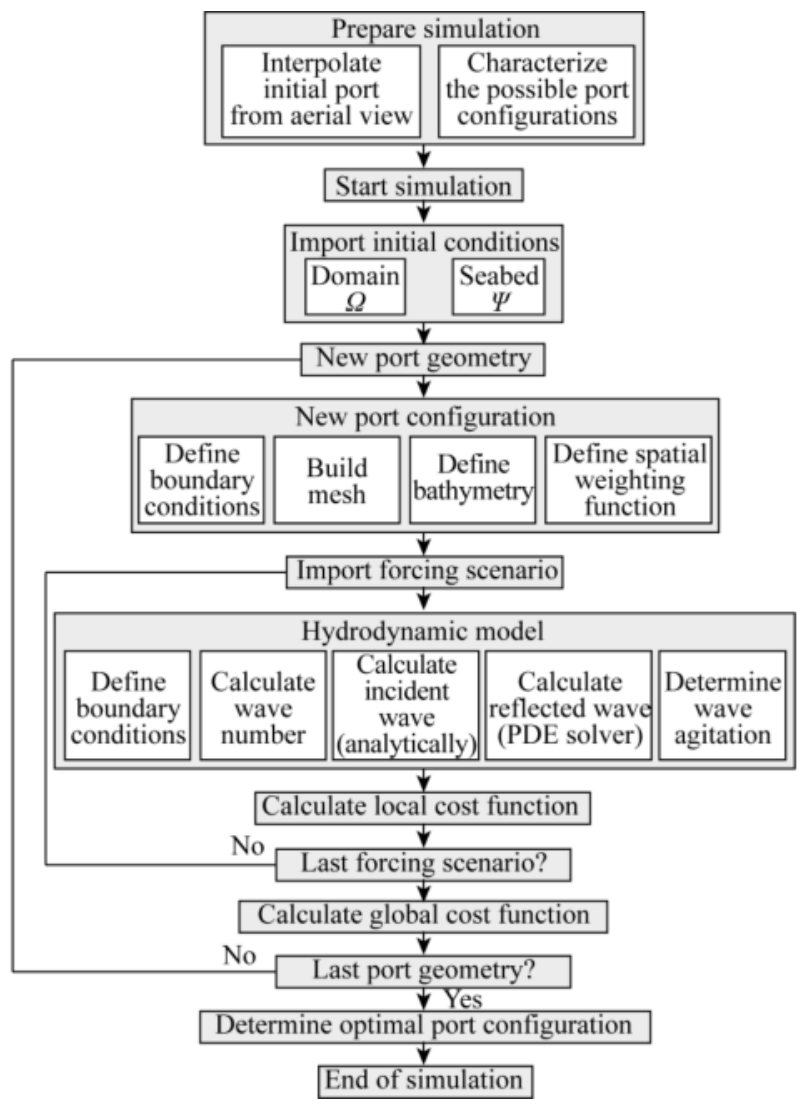

Fig. 2. Numerical strategy applied to the search of the optimal configuration of La Turballe Port. 


\subsection{Managing resonance}

We mentioned the necessity of making sure that the solution of the Helmholtz equation is not an eigenmode of the Laplace operator in the domain as these situations lead to artificially high level of wave energy in the basin and therefore the functional.

Obviously, one cannot imagine computing, at each iteration of an optimization procedure, all the eigenmodes of the Laplace operator for the corresponding domain to make sure that the corresponding energy level is not artificially driven by some resonance phenomena. This would increase dramatically the computation time and is thus opposite to the optimization philosophy.

An alternative method must be set to control resonance efficiently. The method chosen aims to reducing the energy of the port in the case of an energy exceeding a given threshold, here $10^{5} \mathrm{~J}$, which is by far a maximum energy that could be observed in the port. This simple method assumes that any huge amount of energy observed in the port must be due to numerical resonance and therefore must be reduced to secure more realistic results.

Resonance is not often observed in real basin because wave-wave interactions reduce the concentration of energy on one specific frequency, preventing this situation from occurring. However, the hydrodynamical model of this study is based on many superimposed computations of the linear Helmholtz equation forced by monochromatic waves, which does not account for non-linear processes, which explains the possible high energy levels to control.

\section{Application to La Turballe Port}

In this section, we have applied the previous optimization strategy to the port of La Turballe (France). The present work accompanies a more traditional engineering approach in finding the best configuration satisfying all parties involved. Contrarily to the port preciously mentioned, this port does not possess an outlet boundary.

\subsection{Presentation of the port}

Situated along the Atlantic coast in the North-West of France, the port of La Turballe is home to a wide range of maritime activities, including the presence of a marina, numerous fishing facilities as well as a ship repair services. With the increase of these activities and the arrival of others, the department of LoireAtlantique wishes to increase the available surface area of the port. This project accompanies the plan of introducing an offshore wind turbine farm, since its maintenance center is expected to be installed at the La Turballe Port. Its expansion is therefore crucial for the well-being of users of the port. The objective of the project is to expand the port in such a way that the agitation within the port is reduced, thereby reducing the damages to moored boats. Damages caused by excessive agitation within the port include sinking, stranding, colli- sions, and overturning. The aim of this study is to alter the geometry of the port while verifying the following conditions: the surface area of the port must be increased and the agitation of the long waves within the port must be reduced.

Usually, waves customarily arrive nearby a port with some incidence (Wu and Liu, 1985; Booij and Holthuijsen, 1987; Niemeyer et al., 2000). However, in light of the orientation of the port of La Turballe with regard to the mean orientation of the coast and the incoming wave spectrum in this area, no direct waves can enter the port. Furthermore, the large rounded jetty head located at the seaward side of the port entrance reflects any direct incoming waves. It also drives a significant diffraction pattern of any incoming wave forcing that result in the scattering of the wave field towards the shore, but not in the direction of the inner port. This claim is supported by observational data of said port. Therefore, the only source of external energy generating port agitation is not that of the direct wave spectrum, but the energy resulting from the transformation of a part of the wave spectrum into low frequency oscillations at the port entrance. This is the primary reason why the dimensioning of La Turballe is a question of low frequency agitation, and not a problem relative to the direct impact of oceanic waves. To handle this classic situation in harbours, we shoaled the deep water wave conditions towards the port entrance, calculated the energy brought by the waves at this point, and we fed our model directly with an equivalent wave forcing representative of such energetic conditions assuming that the totality of energy associated with the incoming waves is transferred by diffraction and wave spectrum transformation to the port entrance.

\subsection{Setting}

Through classic engineering, it was established that the best course of action was through the installation of a jetty and a mole to produce a bottleneck effect at the entrance of the port. As illustrated in Fig. 3, the jetty extends in an eastwestern direction to form a basin. The mole is attached to the existing structure occupied by parking facilities.

The dimensions of these structures will be determined using optimization theory to ensure minimal agitation with-
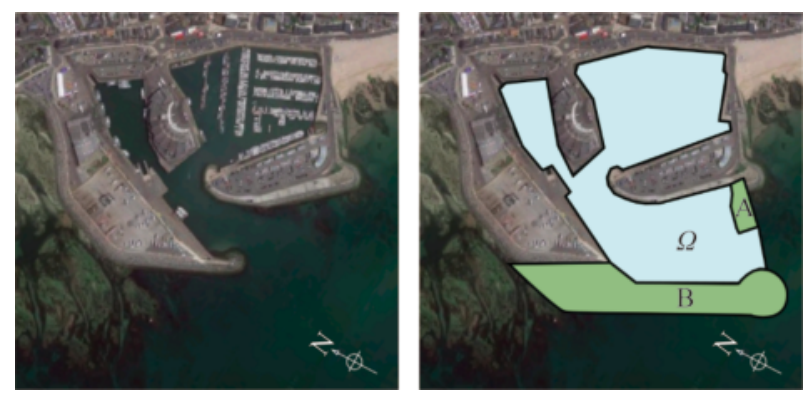

Fig. 3. Illustration of La Turballe Port. Left: The port in its initial state. Right: The port with the two additional structures considered in this study: Mole A and Jetty B. 
in the port. However, certain constraints are imposed in order to guarantee practical results. These constraints include the location of the mole along the existing wall, the dimensions of the mole, the location of the jetty (the extremity must be fixed to the existing port and cannot be installed in waters deeper than $5.5 \mathrm{~m}$ ) and the dimensions of the jetty. The structures must also be sufficiently elevated to be considered impassable by wave overtopping. Each structure is characterized by the dissipation factor $\gamma$ defined in Eq. (9). In order to investigate the most critical scenario, we consider the boundaries to be fully reflective by setting $\gamma=1$.

The bathymetric properties within the port have been observed to be stable, with a depth ranging between $1.5 \mathrm{~m}$ and $3.5 \mathrm{~m}$. We therefore omit the changes to the seabed from the optimization simulation.

In order to conduct this study, we were provided with wave scenarios that were deemed representative of the forcing conditions of the port. These scenarios were given in the form of statistical data of the directional waves (significant wave height, peak period, average direction, etc.) and did not include the probability of occurrence of each scenario. Given the nature of our study which prioritizes the minimization of extreme weather waves (with the introduction of the weighted global cost function), the probability of occurrence of each scenario is irrelevant. These data could not be used in the initial state in the optimization process, because they were attained at different depths surrounding the port. This results in forcing scenarios that cannot be easily compared. The idea is therefore to propagate the wave conditions to the entrance of the port in a classic manner and using a regional wave simulation tool, TELEMAC (Hervouet, 2007; Galland et al., 1991). Certain assumptions involving the nature of the waves and the configuration of the port were made to simplify the calculations, but have no consequences on the results aside from a slight increase in wave agitation. Since this study was conducted for a worstcase scenario, it makes no difference if a little more energy enters the port. This guarantees additional security on the final port configuration.

This process ultimately provided the study with twentyeight different forcing scenarios, representing conditions ranging from fair weather to severe storms. These scenarios are given in the form of three parameters: the frequency, angle and amplitude of the incoming wave $\left(f, \theta, a_{\max }\right)$. These are then plugged into the hydrodynamic model presented in Section 2, noting that $\omega=2 \pi f$, to calculate the wave field over the domain.

By way of illustration, fair weather conditions resemble $\left(f, \theta, a_{\max }\right)=(0.00373,1.47,0.344)$, whereas the more extreme conditions are of the like of $\left(f, \theta, a_{\max }\right)=(0.0261$, $1.25,3.75)$. Some scenarios may exceed the operating limit given by the ship mooring stability conditions (PIANC, 1995). The inclusion of these scenarios ensures port stability, in so far as possible, even in the most extreme forcing scenarios.

\subsection{Parameterization of the port}

For illustrative purposes, we present the search for the optimal configuration of the port using two degrees of freedom only. We define the set of parameters by $\psi=(\alpha, \beta)$, where $\alpha$ represents the width added to Mole A along the existing wall and $\beta$ represents the length of the extension of Jetty B. Fig. 4 illustrates the definitions of $\alpha$ and $\beta$. The choice of these parameters originates from the expertise of classic port engineering, and incorporates the required design constraints. The total width of Mole A must measure between $30 \mathrm{~m}$ and $180 \mathrm{~m}$, whereas the length of the jetty must measure between $350 \mathrm{~m}$ and $550 \mathrm{~m}$.

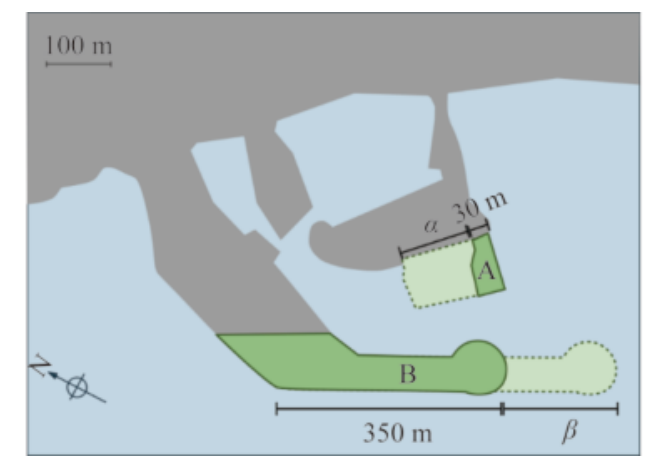

Fig. 4. Illustration of the two degrees of freedom ( $\alpha$ and $\beta)$ used in the search of the optimal solution.

The optimization problem becomes: find $\psi=(\alpha, \beta)$ such that $\mathscr{G}(\psi)$ is minimal and the following constraints are met: $0 \leqslant \alpha \leqslant 150$ and $0 \leqslant \beta \leqslant 200$.

Following the discussion with the industrial experts leading the study of the redesign of La Turballe Port, it was established that the minimization of wave agitation should be concentrated in the south basin of the port. This choice is natural given the dense network of boats and the plan to add a dock and/or wharf to this area. Therefore, the cost function $\mathcal{Y}$, defined by Eq. (11), features the spatial weighting function $\mathscr{P}_{1}$. This function, defined by Eq. (12), is illustrated in Fig. 5 over $\Omega(\psi)$ for a given parameterization $\psi$ of the port.

\section{Results}

In this section, we present the results of the hydrodynamic model presented in Section 2 applied to the port of La Turballe, as well as the results of the optimization calculations.

\subsection{Hydrodynamic simulations}

Fig. 6 illustrates the amplitude of the wave field within the La Turballe Port under moderately severe weather conditions. We observe that the energy originates from the forcing boundary at the entrance of the port. Crest and troughs are observed as the waves propagate throughout the port re- 


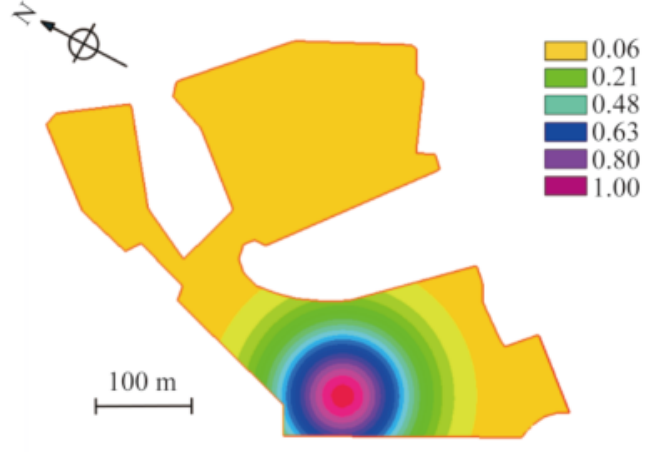

Fig. 5. Spatial weight function $\mathscr{P}_{1}$ defined over $\Omega(\psi)$ for a given parameterization $\psi$ of the La Turballe Port.

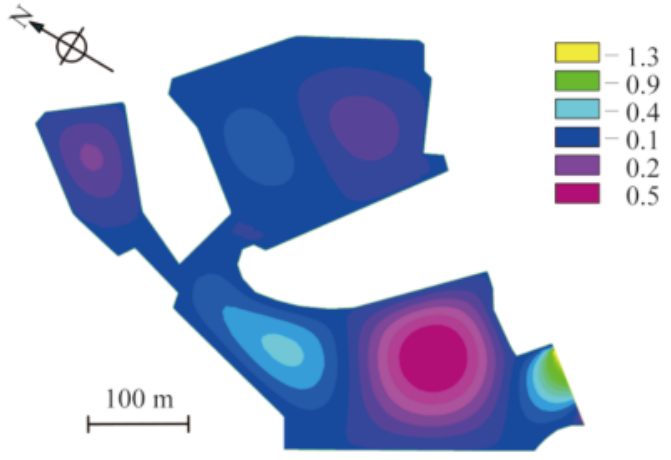

Fig. 6. Oscillation of waves within the port of La Turballe, for a given parameterization of the port. The wave field results from forcing conditions associated with scenario $n=23$ and characterized by $\left(f, \theta, a_{\max }\right)=$ $(0.0186,1.25,1.50)$.

acting to the solid borders and show signs of seiche-like behavior.

\subsection{Optimization simulations}

The local cost function associated with forcing scenario $n=4$ is given in Fig. 7. Here the forcing conditions, given by $\left(f, \theta, a_{\max }\right)=(0.0261,1.47,0.172)$, depict fair weather conditions. Several local minima are observed, which demonstrate the necessity of adopting an optimizing program capable of detecting a global minimum. Otherwise,

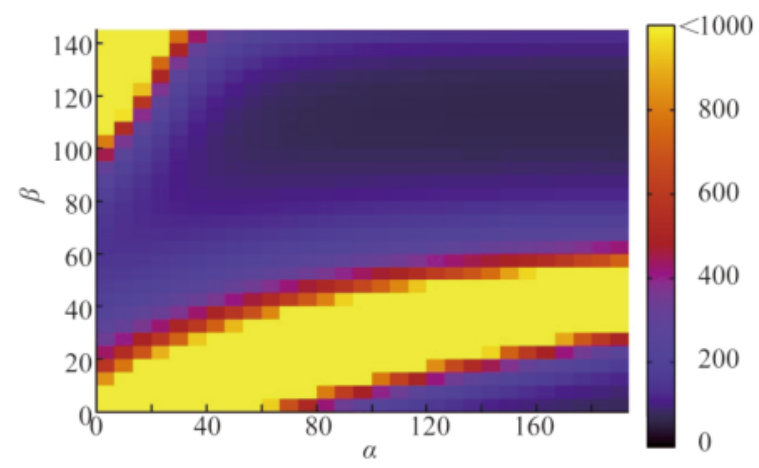

Fig. 7. Local cost function $\mathscr{G}(\alpha, \beta)$ with only one forcing scenario considered and several local minima located at the dark purple regions of the graph. problems may occur, especially if the number of design parameters is increased.

Fig. 8 represents the values of the cost function $\mathscr{I}$ defined by Eq. (13) with respect to the parameters $\alpha$ and $\beta$. The minimum of $\mathscr{G}$ is marked by a cross and is located away from the borders. Despite the presence of several local minima in one forcing scenario, the linear combination of scenarios that form the global cost function leads to a unique global minimum. This may differ depending on the choice of parameters $\psi$. We deduce that the study of the optimal solution with two degrees of freedom provides the following results: $\alpha^{*}=90$ and $\beta^{*}=107$. We notice great variability of the solution over the $\alpha$ axis, whereas over $\beta$, the function is relatively independent. This suggests that the decrease of wave agitation within the port is greatly influenced by dimensions of the mole and less so by the extension of the jetty.

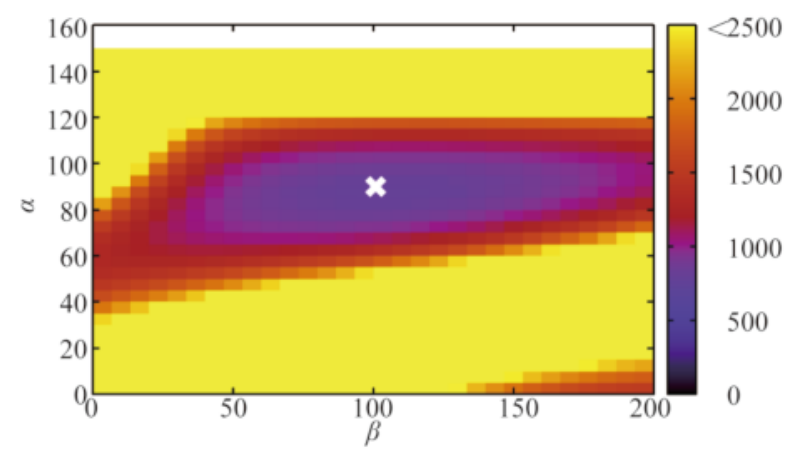

Fig. 8. Cost function $\mathscr{G}(\alpha, \beta)$. The optimal solution is indicated by a cross. In this case, we have a unique global optimum and a local minimum.

The corresponding configuration is given in Fig. 9. The jetty is extended by $107 \mathrm{~m}$ and the mole is widened by $90 \mathrm{~m}$.

\subsection{Energetic state of the port}

Fig. 10 represents the values of the local cost function $I_{n}$ with regard to the 28 different forcing scenarios here represented on the $X$-axis by their reference number. Each point plotted on the figure corresponds to the value of the function $\mathscr{Y}_{n}$ calculated for a given configuration of the port and a given forcing scenario $n$.

We therefore have a graphical representation of the set of the values of the functional $\mathscr{I}_{n}$ for all scenarios and all configurations explored by optimization simulation. Along a vertical line (i.e. for a given forcing scenario), we see the values of $\mathscr{I}_{n}$ for the scenario $n$, according to different configurations. The lowest point corresponds to the local optimal configuration for this forcing scenario. The red marker indicates the energy $\mathscr{I}_{n}$ associated with the configuration $\left(\alpha^{*}, \beta^{*}\right)$ for each of the forcing scenarios. For each of the 28 cases, this marker is situated in the lower range of the possible values, showing that the minimum of the global cost function $\mathscr{G}$ is equivalent to the minimum of each of the local cost functions $\mathscr{I}_{n}$. 


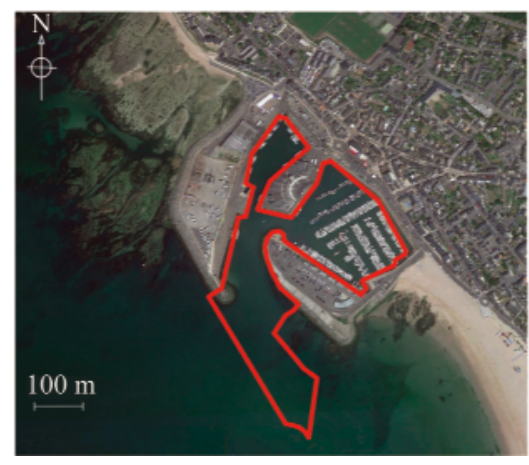

Fig. 9. Analysis of the efficiency of the global optimal solution, with the parameters being limited to two degrees of freedom $(\alpha, \beta)$ and forced by the 28 scenarios. The diagram represents the variation of the global cost function $\mathscr{G}$ with regard to the forcing scenario and for each possible port configurations (red markers correspond to the values of $\mathscr{G}$ associated with each parameterization of the port, green corresponds to the values of $\mathscr{I}$ associated with the global optimal solution).

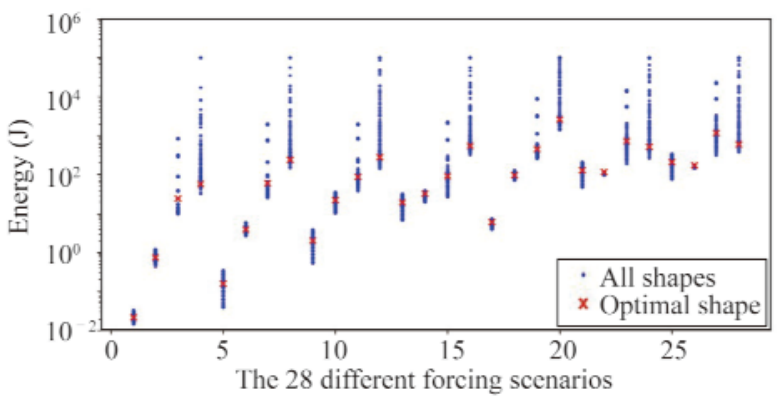

Fig. 10. Inner border (red) of the optimal configuration of the port, resulted from the minimization of energy. We observe an extended jetty and a wide mole.

In each case, the optimal solution has significantly reduced the quantity of energy within the port. Let us take the example of Scenario 4. The energy within the port can potentially be in the order of $105 \mathrm{~J}$; this corresponds to a wave height of $4.5 \mathrm{~m}$. The optimal configuration, marked by a red point, results in an energy of $58 \mathrm{~J}$, which corresponds to a wave height of $0.12 \mathrm{~m}$. This reduction of $4.38 \mathrm{~m}$ in wave height demonstrates the efficiency of the optimization model in determining the best configuration of the port. As the lowest possible energy of the port for Scenario 4 is $33 \mathrm{~J}$, which corresponds to a wave height of $0.082 \mathrm{~m}$, we can conclude that the optimization model provides a quasi-optimal solution for this forcing scenario. This reasoning can be applied to each forcing scenario, which leads us to conclude that the optimization model provides a solution to reduce the agitation in the port for all types of forcing conditions.

\section{Discussion}

Despite finding an optimal solution to reduce the agitation of the water within the port whilst increasing its exploitable surface area, the solution may be deemed unfit if it has an undesirable effect on its environment. A study has therefore been conducted so as to determine the morphological impact of the new configuration on the surrounding shoreline. Depending on the results, the optimal solution may or may not be retained.

\subsection{GenCade model}

The study of the morphological effect of the newly designed port was modeled by GenCade (Hanson et al., 2011), a numerical model developed by the CIRP and the Regional Sediment Management Program which combines the engineering processes of GENESIS (Hanson and Kraus, 1989) and the long-term, regional transport processes of Cascade (Connell et al., 2007).

This model simulates the evolution of the shoreline and the transport of sand over time. Capable of incorporating different engineering structures, such as jetties, breakwaters, and seawalls as well as other activities such as beach nourishment, this model is often used to determine the consequences of introducing man-made structures to the coastal environment. Examples of this model being applied to the study of shoreline dynamics include Frey et al. (2012b), Schrader et al. (2016) and Frey et al. (2012a).

\subsection{Setup}

GenCade was applied to an area surrounding La Turballe Port, covering a distance of over $5 \mathrm{~km}$. Two set of simulations were conducted in order to compare the evolution of the shoreline with and without the jetty and mole studied in Section 4. Fig. 11 shows the two initial shoreline conditions used in the simulation. The blue line indicates the shoreline with the newly determined jetty and mole whereas the red delimits the shoreline in its actual state. Characteristic forcing conditions of the area were applied over the duration of 30 years.

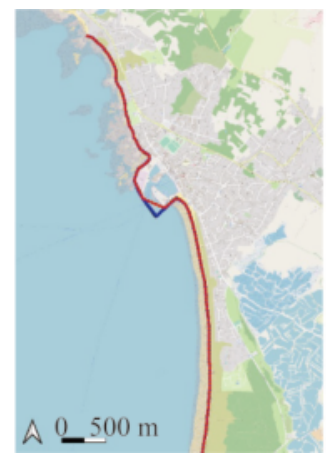

Fig. 11. Two initial shoreline conditions of La Turballe Port.

The aim of this simulation was to confirm that the redesign of the port has little influence on the surrounding shoreline in long term. We cannot compare simulation with field data since the simulations are purely forecasts. We set the Gencade model with typical parameters for the beaches surrounding La Turballe, and a comparison was performed 
with and without the port transformation. The modeling of the long term shoreline dynamics is relative on account of Gencade using a purely linear formalism (Pelnard Considère equation, cf. Pelnard-Considère,1956), but does provide a trend.

\subsection{Results}

Fig. 12 shows the results of two simulations conducted by GenCade, in the area surrounding the port. Beyond this area, no changes to the shoreline are observed, arguing that the new layout of the port only impacts the surrounding shoreline within a $150 \mathrm{~m}$ radius, and no long term impact can be expected.

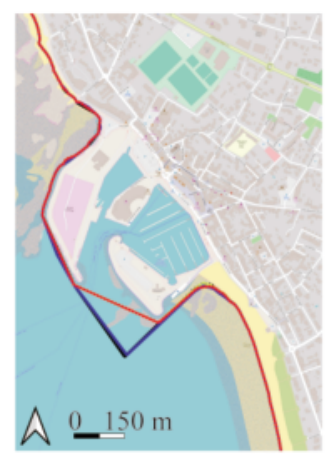

Fig. 12. Results of the long term morphodynamic simulation with and without the proposed structures. The simulations without the redesign of the port are given by the dotted lines: yellow is the initial shoreline and red is the shoreline after the 30 -year simulation. The simulations incorporating the redesign of the port are given by the solid lines: black is the initial shoreline and blue is the shoreline after the 30 -year simulation.

In the vicinity of the port, two areas of discrepancy can be observed. The first is located in the area where the beach reaches the port. However, the difference between the two configurations does not exceed $15 \mathrm{~m}$ over the 30 -year period. The second zone of interest is situated on the border of the jetty exposed to the ocean waves. Here, a difference of several meters is observed. Given that this border is a solid wall and cannot vary over time, the discrepancy can be explained as the result of numerical inaccuracy against such solid boundary condition. Given the results provided by GenCade, we can conclude that redesigning the port to include a jetty and mole does not impact the shoreline dynamics of the surrounding area.

\section{Conclusions}

The motivation behind this numerical model is not to determine the ultimate port design, which would require extensive verification and validation procedures, but to provide a description of a new rapid and cost effective optimization tool, with an application to La Turballe Port. This tool was designed to accompany classical engineering approaches and should not be the sole component of the port design study; the Verification and Validation (V\&V) component is undeniably required when approving a new port design. However, this is beyond the scope of the numerical work presented here. In this paper, we demonstrate that the rapidity and adaptiveness of the model allows the engineers in charge to focus on practical solutions efficiently. They should subsequently perform a thorough V\&V of the considered port configurations.

The final redesign of the port promoted by the procedure satisfies the constraints imposed, is a good solution for any incoming forcing considered and thus delineates the best solution ever. This solution is consistent with classic engineering. Despite the calculations being centered on the minimization of wave agitation and the increase of exploitable surface area, the optimal solution also has the advantage of having no significant impact on the surrounding shoreline dynamics. This work demonstrates that numerical optimization may be quick and efficient in the identification of port solutions consistent with classic engineering even in the context of complex problems.

\section{Acknowledgements}

This work describes a full textbook case of port engineering redesigned by optimization theory combined with a posteriori management of an environmental question. The concepts and a comprehensive methodology are presented on the very classic port of La Rochelle; then a realistic application is performed for the redesign of La Turballe Port. The optimal solution presented in Section 4 where an elongated jetty and a widened mole is preferred is determined by optimization theory.

\section{Right and permissions}

Open Access This article is licensed under a Creative Commons Attribution 4.0 International License, which permits use, sharing, adaptation, distribution and reproduction in any medium or format, as long as you give appropriate credit to the original author(s) and the source, provide a link to the Creative Commons licence, and indicate if changes were made. The images or other third party material in this article are included in the article's Creative Commons licence, unless indicated otherwise in a credit line to the material. If material is not included in the article's Creative Commons licence and your intended use is not permitted by statutory regulation or exceeds the permitted use, you will need to obtain permission directly from the copyright holder. To view a copy of this licence, visit http://creativecommons.org/licenses/by/4.0/.

\section{References}

Alises, A., Molina, R., Gómez, R., Pery, P. and Castillo, C., 2014. Overtopping hazards to port activities: Application of a new methodology to risk management (port risk management tool), Reliability Engineering \& System Safety, 123, 8-20.

Allsop, N.W.H. and Hettiarachchi, S.S.L., 1989. Wave Reflections in Harbours: Design, Construction and Performance of Wave Absorbing Structures, HR Wallingford, Oxford.

Berkhoff, J.C.W., 1972. Computation of combined refraction - diffrac- 
tion, Proceedings of the 13th International Conference on Coastal Engineering, American Society of Civil Engineers, Vancouver.

Booij, N. and Holthuijsen, L.H., 1987. Propagation of ocean waves in discrete spectral wave models, Journal of Computational Physics, 68(2), 307-326.

Bouharguane, A., Azerad, P., Bouchette, F., Marche, F. and Mohammadi, B., 2010. Low complexity shape optimization \& a posteriori high fidelity validation, Discrete \& Continuous Dynamical SystemsB, 13(4), 759-772.

Bouharguane, A. and Mohammadi, B., 2012. Minimisation principles for the evolution of a soft sea bed interacting with a shallow sea, International Journal of Computational Fluid Dynamics, 26(3), 163-172.

Castillo, E., Losada, M.A., Mínguez, R., Castillo, C. and Baquerizo, A., 2004. Optimal engineering design method that combines safety factors and failure probabilities: Application to rubble-mound breakwaters, Journal of Waterway, Port, Coastal, and Ocean Engineering, 130(2), 77-88.

Connell, K.J., Larson, M. and Kraus, N.C., 2007. Morphologic modeling of multiple barrier island breaches for regional application, Proceedings to the Sixth International Symposium on Coastal Engineering and Science of Coastal Sediment Process, (ASCE), 2011-2073.

Cornett, A. and Baker, S., 2018. Value of 3D physical modeling in harbor design - gateway harbor Chicago case study, Proceedings of the 34th PIANC World Congress, PIANC, Panama.

Frey, A.E., Rosati III, J., Connell, K.J., Hanson, H. and Larson, M., 2012a. Modeling alternatives for erosion control at Matagorda county, Texas, with Gencade, Proceedings to the 33rd International Conference on Coastal Engineering 2012, Coastal Engineering Research Council, Santander, Spain.

Frey, A.E., Munger, S., Williams, G.L., Wutkowski, M.J. and Conner, K.B., 2012b. GenCade Application at Onslow Bay, North Carolina, U.S. Army Engineer Research and Development Center, Coastal and Hydraulics Laboratory, Vicksburg.

Galland, J.C., Goutal, N. and Hervouet, J.M., 1991. TELEMAC: A new numerical model for solving shallow water equations, $A d-$ vances in Water Resources, 14(3), 138-148.

Groenveld, R., 1983. Harborsim, A Generally Applicable Harbour Simulation Model, TU Delft, Section Hydraulic Engineering, Delft.

Hanson, H., Connell, K.J., Larson, M., Kraus, N.C., Beck, T.M. and Frey, A.E., 2011. Coastal evolution modeling at multiple scales in regional sediment management applications, Proceedings of Coastal Sediments 2011 Conference, World Scientific, Miami, pp. 1920-1932.

Hanson, H. and Kraus, N., 1989. Genesis: Generalized model for simulating shoreline change, Proceedings of the 30th International Conference on Coastal Engineering, pp. 3762-3773.

Harris, G.A., Anderson, M., Schroer, B.J., Landrum, B. and Möller, D.P.F., 2009. A simulation model for determining container throughput at an expanding seaport, Proceedings of the 6th Vienna International Conference on Mathematical Modeling MATHMOD2009, Vienna, Austria.

Hervouet, J.M., 2007. Hydrodynamics of Free Surface Flows: Modelling with the Finite Element Method, John Wiley \& Sons, Ltd., New York.

Hewitt, C.L. and Martin, R.B., 2001. Revised Protocols for Baseline Port Surveys for Introduced Marine Species: Survey Design, Sampling Protocols and Specimen Handling, CSIRO Marine Research, Hobart.

Isebe, D., Azerad, P., Bouchette, F., Ivorra, B. and Mohammadi, B., 2008a. Shape optimization of geotextile tubes for sandy beach protection, International Journal for Numerical Methods in Engineering, 74(8), 1262-1277.

Isebe, D., Azerad, P., Mohammadi, B. and Bouchette, F., 2008b. Optimal shape design of defense structures for minimizing short wave impact, Coastal Engineering, 55(1), 35-46.

Isebe, D., Bouchette, F., Mohammadi, B., Azerad, P., Lambert, A., Bujan, N., Grasso, F. and Michallet, H., 2008c. Une nouvelle approche pour la protection des plages: Applications àla plage du lido de sète, Proceedings of the Xemes Journées Génie Civil-Génie Cotier, Revue Paralia, Sophia-Antipolis, pp. 263-272. (in French)

Ivorra, B., Isèbe, D. and Mohammadi, B., 2005. Optimisation globale à complexité réduite: application à divers problèmes industriels, Proceedings of the 7e Colloque National en Calcul des Structures, CSMA, Giens, France. (in French)

Jahren, C.T. and Ishii, S., 1995. Emergency ferry landing design, Journal of Waterway, Port, Coastal, and Ocean Engineering, 121(4), 216-222.

Joubert, F.J. and Pretorius, L., 2020. Design and construction risks for a shipping port and container terminal: Case study, Journal of Waterway, Port, Coastal, and Ocean Engineering, 146(1), 05019003.

Li, P.F. and Zhou, X.J., 2015. Mechanical behavior and shape optimization of lining structure for subsea tunnel excavated in weathered slot, China Ocean Engineering, 29(6), 875-890.

Lillycrop, L.S. and Briggs, M.J., 1992. Capabilities in Harbor Design and Monitoring: A Case Study, US Army Engineer Waterways Experiment Station (WES), Coastal Engineering Research Center (CERC), Vicksburg, Mississippi, pp. 11.

McCartney, B.L., 1985. Floating breakwater design, Journal of Waterway, Port, Coastal, and Ocean Engineering, 111(2), 304-318.

Mohammadi, B. and Bouchette, F., 2014. Extreme scenarios for the evolution of a soft bed interacting with a fluid using the value at risk of the bed characteristics, Computers \& Fluids, 89, 78-87.

Mohammadi, B. and Bouharguane, A., 2011. Optimal dynamics of soft shapes in shallow waters, Computers \& Fluids, 40(1), 291-298.

Mohammadi, B. and Pironneau, O., 2009. Applied Shape Optimization for Fluids. Numerical Mathematics and Scientific Computation, second ed., Oxford University Press, Oxford.

Mohammadi, B. and Saiac, J.H., 2003. Pratique de la Simulation Numérique, Dunod. (in French)

Niemeyer, H.D., Kaiser, R. and Weiler, B., 2000. Design wave evaluation for coastal protection structures in the Wadden Sea, Proceedings of the 6th International Workshop on Wave Hindcasting and Forecasting, Environment Canada, Monterey.

Pachakis, D. and Kiremidjian, A.S., 2003. Ship traffic modeling methodology for ports, Journal of Waterway, Port, Coastal, and Ocean Engineering, 129(5), 193-202.

Pelnard-Considère, R., 1956. Essai de théorie de l'évolution des Formes de Rivage en Plages de Sable et de Galets, Société hydrotechnique de France, Paris, pp. 289-298. (in French)

PIANC, 1995. Criteria for Movements of Moored Ships in Harbours: A Practical Guide, PIANC, Brussel.

Rabinovich, A.B., 2017. Seiches and harbor oscillations, in: Kim, Y.C. (ed.), Handbook of Coastal and Ocean Engineering, World Scientific Publishing Co Pte Ltd., Singapore, pp. 243-286.

Saghi, H., Ning, D.Z., Cong, P.W. and Zhao, M., 2020. Optimization of baffled rectangular and prismatic storage tank against the sloshing phenomenon, China Ocean Engineering, 34(5), 664-676.

Sawicki, A., Kulczykowski, M., Robakiewicz, W., Mierczyński, J. and Hauptmann, J., 1998. New type of bottom protection in harbors- 
design method, Journal of Waterway, Port, Coastal, and Ocean Engineering, 124(4), 208-211.

Schrader, M.H., Douglass, E.C. and Lillycrop, L.S., 2016. Regional Sediment Management Strategies for the Vicinity of st. Augustine Inlet, st. Johns County, Florida, U.S. Army Engineer Research and Development Center, Coastal and Hydraulics Laboratory, Vicksburg.

Smith, C.D., 1988. Outlet structure design for conduits and tunnels, Journal of Waterway, Port, Coastal, and Ocean Engineering, 114(4), 503-515.

Tian, Z.L., Sun, Z.C., Liang, S.X. and Wang, X.G., 2018. Inverse calculation of wave-absorbing structure dimensions based on extended ANFIS model, China Ocean Engineering, 32(5), 501-513.

Trbojevic, V.M. and Carr, B.J., 2000. Risk based methodology for safety improvements in ports, Journal of Hazardous Materials, 71(1-3), 467-480
Wang, D.T., Ju, L.H., Zhu, J.L., Wang, Z., Sun, T.T. and Chen, W.Q., 2017. Experimental study on mean overtopping of sloping seawall under oblique irregular waves, China Ocean Engineering, 31(3), 350-356.

Wang, G., Zheng, J.H., Liang, Q.H., Zhang, W. and Huang, C., 2015. Theoretical analysis of harbor resonance in harbor with an exponential bottom profile, China Ocean Engineering, 29(6), 821-834.

$\mathrm{Wu}$, C.S. and Liu, P.L.F., 1985. Finite element modeling of nonlinear coastal currents, Journal of Waterway, Port, Coastal, and Ocean Engineering, 111(2), 417-432.

Wu, G.W., Wu, H., Wang, X.Y., Zhou, Q.W. and Liu, X.M., 2018. Tidal turbine array optimization based on the discrete particle swarm algorithm, China Ocean Engineering, 32(3), 358-364.

Zhang, W.C., Liu, H.X., Zhang, L. and Zhang, X.W., 2016. Hydrodynamic analysis and shape optimization for vertical axisymmetric wave energy converters, China Ocean Engineering, 30(6), 954-966. 